\title{
Effect of neighboring cells on cell stiffness measured by optical tweezers indentation
}

\author{
Muhammad S. Yousafzai \\ Giovanna Coceano \\ Alberto Mariutti \\ Fatou Ndoye \\ Ladan Amin \\ Joseph Niemela \\ Serena Bonin \\ Giacinto Scoles \\ Dan Cojoc
}




\title{
Effect of neighboring cells on cell stiffness measured by optical tweezers indentation
}

\author{
Muhammad S. Yousafzai, ${ }^{a, b, c}$ Giovanna Coceano, ${ }^{a, b}$ Alberto Mariutti, ${ }^{c}$ Fatou Ndoye, ${ }^{\mathrm{d}, \mathrm{e}}$ Ladan Amin, ${ }^{\mathrm{f}}$ \\ Joseph Niemela, ${ }^{d}$ Serena Bonin, ${ }^{9}$ Giacinto Scoles, ${ }^{c}$ and Dan Cojoc ${ }^{a, *}$ \\ Institute of Materials (IOM-CNR), Area Science Park, Basovizza, S.S. 14, Km 163.5, 34149 Trieste, Italy \\ bUniversity of Trieste, PhD School of Nanotechnology, Via Alfonso Valerio 2, Trieste 34127, Italy \\ 'University of Udine, Department of Biological and Medical Science, Santa Maria della Misericordia Hospital, Piazzale Kolbe 4, Udine 33100, Italy \\ ${ }^{\mathrm{d} T}$ The Abdus Salam International Centre for Theoretical Physics (ICTP), Via Beirut 6, Trieste 34149, Italy \\ ${ }^{e}$ Cheikh Anta Diop University, Department of Physics, Dakar 5005, Senegal \\ fInternational School for Advanced Studies (SISSA), Neuroscience Area, Via Bonomea 265, Trieste 34136, Italy \\ gUniversity of Trieste, Department of Medical Sciences, Cattinara Hospital, Strada di Fiume 447, Trieste 34149, Italy
}

\begin{abstract}
We report on the modification of mechanical properties of breast cancer cells when they get in contact with other neighboring cells of the same type. Optical tweezers vertical indentation was employed to investigate cell mechanics in isolated and contact conditions, by setting up stiffness as a marker. Two human breast cancer cell lines with different aggressiveness [MCF-7 (luminal breast cancer) and MDA-MB-231 (basal-like breast cancer)] and one normal immortalized breast cell line HBL-100 (normal and myoepithelial) were selected. We found that neighboring cells significantly alter cell stiffness: MDA-MB-231 becomes stiffer when in contact, while HBL-100 and MCF-7 exhibit softer character. Cell stiffness was probed at three cellular subregions: central (above nucleus), intermediate (cytoplasm), and near the leading edge. In an isolated condition, all cells showed a significant regional variation in stiffness: higher at the center and fading toward the leading edge. However, the regional variation becomes statistically insignificant when the cells were in contact with other neighboring cells. The proposed approach will contribute to understand the intriguing temporal sequential alterations in cancer cells during interaction with their surrounding microenvironment. ๑ 2016 Society of Photo-Optical Instrumentation Engineers (SPIE) [DOI: 10.1117/1.JBO.21.5.057004]
\end{abstract}

Keywords: optical tweezers; cell stiffness; indentation; cancer; cell-cell interaction; microenvironment.

Paper 150853LR received Dec. 21, 2015; accepted for publication Apr. 25, 2016; published online May 27, 2016.

\section{Introduction}

Cancer has been extensively studied to unveil its causes and to develop therapeutic tools for cure and prevention. ${ }^{1,2}$ There are multitudes of factors of its occurrence but the major, known, factors lie in the genetic mutations, which lead to aberrant signaling pathways, causing these cells to become malignant. ${ }^{2}$ Most studies have focused on cancer genomics to unravel malignant transformation and cancer invasion; nonetheless, it has emerged that genetics and molecular biology alone have not been able to address the problem completely. ${ }^{3-5}$ Therefore, efforts have been made to approach cancer from a mechanical point of view, which correlates biological processes with cell mechanics. ${ }^{6-12}$ Cells constantly sense mechanical forces from their microenvironment [extracellular matrix (ECM) and neighboring cells] that play a pivotal role in cell functions. ${ }^{7,13}$ Mechanical properties of cancer microenvironment are crucial, as they may have a role in cancer initiation, promotion, or they may cause the cancer to revert back. ${ }^{14,15}$ At the very early stage, when a cell turns into cancerous, a very strong interplay between microenvironment and the cell gets initiated, leading to cell cytoskeleton alterations, ECM stiffening, tumor progression, and metastasis. ${ }^{16,17}$
In the past decade, the mechanical interaction of a cell with its microenvironment has garnered much attention and witnessed considerable progress. ${ }^{18}$ The microenvironment is the driving element in cell proliferation, ${ }^{19}$ viscoelasticity, ${ }^{4,7,20}$ functionality, ${ }^{21}$ and cell signaling. ${ }^{22,23}$ The mechanical interaction of the cell with the surrounding microenvironment is a blend of cell-matrix and cell-cell interactions. Both of these processes have prime importance for the fundamental understanding of metastasis and cell behavior in malignant conditions. This article is focused on cell-cell interaction dynamics for breast cancer cells. Cell-cell interactions are complex and include cell-cell interactions in normal tissues, primary tumor, interactions during transit at the metastasis stage, and secondary tumor site. The cell-cell mechanical interaction has variant spatial and temporal tendencies and the precise knowledge of these interactions is still lacking. Initiation, detachment, and organ-specific affinity of cancer cells to host cells in terms of mechanical interaction can reveal deeper understanding of cancer progression and metastasis.

Many studies have highlighted cell-cell interactions in terms of adhesion and other chemophysical properties, but few studies have been devoted to their mechanistic nature. The interaction between MDA-MB-231 cells and noncancerous Madin-Darby canine kidney (MDCK) epithelial cells has been studied in 
time-lapse three-dimensional (3-D) configuration by Ivers et al., ${ }^{24}$ demonstrating that microenvironment has a strong impact on the growth and dynamics of cancer cells by stressing the organ-specific affinity of cancer cells to host cells. Using the optical tweezers (OT) pulling method, Hu et al. ${ }^{25}$ categorized stroma-cell interactions as tightly adherent, loosely adherent, and freely suspended. They also observed dynamic signaling pathways during cell-cell interactions coupling OT to fluorescence microscopy. ${ }^{26}$ The effect of neighboring cells on cell stiffness was recently investigated for human mammary epithelial cells, representing four different phases of cancer progression, by means of atomic force microscopy (AFM). ${ }^{27}$ This work reports that cell-cell contact strongly influences the cell stiffness of normal cells, while it has little effect on the cancer cells. In another recent study, Kamińska et al. ${ }^{28}$ have reported a body of evidence indicating that the interaction between normal and neoplastic cell contributes to tumor growth.

In this study, we employed OT to measure cell stiffness by using a similar approach to that of AFM cell indentation. AFM and OT indentation regimes (force and loading rate) are very different, making these techniques complementary. As shown in some recent papers, ${ }^{29,30}$ the use of both OT and AFM enables the separation of the viscous and elastic components and provides a more robust method to investigate cell stiffness. We investigated the cell stiffness of three types of human breast cells (non-neoplastic, tumorigenic, and metastatic) in isolated and cell-cell contact conditions. The key results of our work are as follows:

1. For all the three cell lines, the stiffness of the cell in isolated condition is higher at the nucleus region and decreases toward the cell edge, confirming recent AFM results reported for similar cell lines..$^{30,31}$

2. Cell neighboring has a significant effect on the stiffness of metastatic cells; they become stiffer than their counterpart in isolated conditions. This change is confirmed both for the region over the nucleus and regions between the nucleus and the cell edge. The other two cell lines are less sensitive to the cell-cell contact condition. This result is stressing the importance of cell microenvironment when defining cell stiffness.

\section{Materials and Methods}

\subsection{Cells Sample Preparation}

MDA-MB-231, MCF-7, and HBL-100 cell lines (ATCC numbers HTB-26, HTB-22, and HTB-124, respectively) were cultured in adhesion using low glucose Dulbecco's modified Eagle medium with L-glutamine (MDA-MB-231 and MCF-7) or Roswell Park Memorial Institute 1640 medium with L-glutamine (HBL-100), supplemented with $10 \%(\mathrm{v} / \mathrm{v})$ fetal calf serum, $50 \mathrm{IU} / \mathrm{ml}$ of penicillin-streptomycin, and $1 \mathrm{mM}$ gentamycin. Cell cultures were maintained in $25-\mathrm{cm}^{2}$ flasks at $37^{\circ} \mathrm{C}$ in $5 \% \mathrm{CO}_{2}$. Cell splitting was performed every 2 to 3 days, as soon as the cultures reached confluence, using 1:10 diluted $0.05 \%$ trypsin-EDTA. The day before the experiments, the cells were seeded overnight on $18-\mathrm{mm}$ glass coverslips at a density of $10 \times 10^{4}$ cells $/ \mathrm{ml}$ and $15 \times 10^{4}$ cells $/ \mathrm{ml}$ in $2 \mathrm{ml}$ of medium. Before starting the experiment, cells were washed three times in phosphate-buffered saline (PBS) and rinsed with medium before every measurement session. All reagents for cell culture were purchased from Gibco
Lifetechnology; cell culture flasks and petri dishes were purchased from Sigma-Aldrich. ${ }^{32}$

\subsection{Optical Tweezers Indentation}

We employed a modified Thorlabs OKBT modular setup for force measurement by the vertical cell indentation technique, previously described by Yousafzai et al. ${ }^{33}$ Briefly, an infrared (IR) laser (single-mode Yb fiber laser YLM-5, $1064 \mathrm{~nm}$, max $5 \mathrm{~W}$, IPG Photonics $\mathrm{GmbH}$ ) is focused into the sample chamber through Nikon $100 \times$ lens [NA 1.25 oil immersion, working distance (WD) 0.3] to produce a stable trap (Fig. 1). During force measurements, the cells cultured on a coverslip are kept at physiological temperature $\left(37^{\circ} \mathrm{C}\right)$ using a temperature controller unit (RS components). The sample is imaged through CMOS camera (Thorlabs-DCC1240C). The trapped bead is positioned above the cell using a nanopiezo cube, PS, (Thorlabs, NanoMax 3-axis flexure stage), allowing to control the sample displacement with $<5$-nm resolution. The position of the trapped bead is monitored using back focal plane interferometry ${ }^{34}$ of the IR beam scattered by the trapped bead and collected by a second microscope lens (Nikon 10×, NA 0.25, WD 7). The interference pattern is imaged by an additional convergent lens onto the quadrant photo detector (Thorlabs, PDQ80A, detector size $7.8 \mathrm{~mm}$ ), which senses the lateral and axial displacement of the trapped bead. A laser power of $500 \mathrm{~mW}$ was used, which corresponds to $25 \mathrm{~mW}$ at

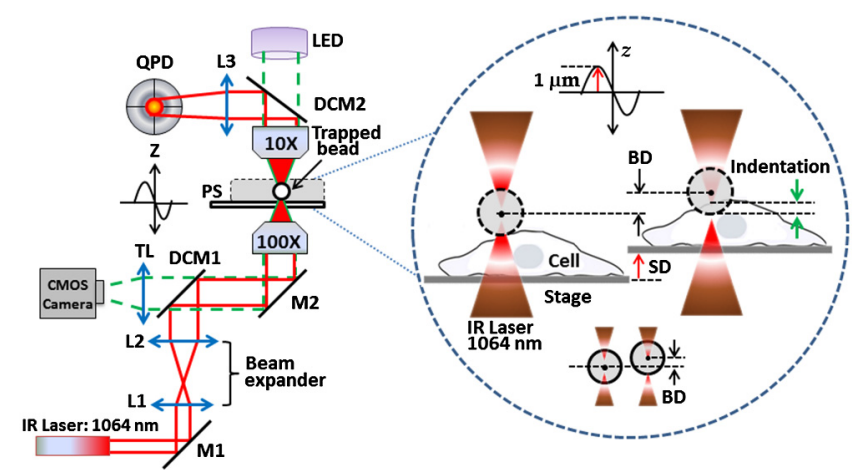

(a)

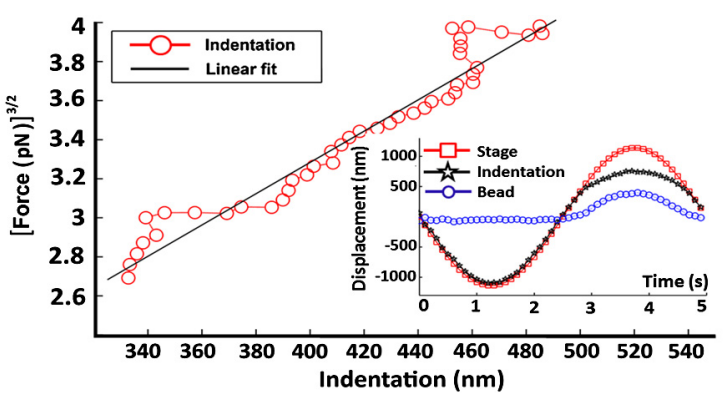

(b)

Fig. 1 (a) OT indentation and force measurement setup. IR trapping laser path (solid line) and bright-field imaging path (dashed line). QPD, quadrant photodiode; PS, 3-axis nanopiezo stage; TL, tube lens; L1 to L3, convergent lenses; M1 to M3, mirrors; and DCM, 1-2 dichroic mirrors. Inset: The cell is positioned below the trapped bead and the stage is moved up by stage displacement (SD). The cell interacts with the bead displacing it by bead displacement (BD) and causing the cell indentation. (b) Linearized force-indentation plot for elastic modulus calculation. Inset: By subtracting BD from SD, indentation is obtained. 
the sample plane and was shown to be not damaging the cells and provide stable trap with a stiffness of $0.015 \mathrm{pN} / \mathrm{nm}$. The cells were indented vertically by moving the cell holder, coupled with PS, against the trapped bead by a sinusoid signal (amplitude $A=1 \mu \mathrm{m}$ and one period $T=5 \mathrm{~s}$ with frequency of $0.2 \mathrm{~Hz}$ ) as shown in Fig. 1, and the displacement of the bead in the trap was acquired at a $10-\mathrm{KHz}$ sampling frequency.

\subsection{Cell Site Selection and Cell Neighboring}

Cells are indented at three locations, at the center above the nucleus (L1), out of the cell nucleus (L2), and near the leading edge (L3), as shown in Fig. 2. Each location was indented at least two times and the results were averaged for each location.

All three cell lines were cultured on the same type of glass coverslips, which act as a hard substrate to the cells (approximately $70 \mathrm{GPa}$ of stiffness). Since the cell senses its surrounding, to understand the effects of neighboring cells on the mechanical properties of the cell under study, the cells were cultured with varying densities (Sec. 2.1) to get cell-cell contact [Fig. 2(b)]. An isolated cell is not touching any other cell, while a connected cell has at least two connecting sites with the neighboring cells. The surrounding cells act as mechanical cage to the cell under study.

\subsection{Cell Stiffness Calculation}

We investigated the elastic modulus of the three cell lines at three different locations of the cell (Sec. 2.3) using OT vertical indentation having a trap stiffness of $0.015 \mathrm{pN} / \mathrm{nm}$. We used $3-\mu \mathrm{m}$ silica beads as the handle to indent cells by moving the stage (cell) against the bead in a sinusoidal $(T=5 \mathrm{~s}$ and $A=$ $1 \mu \mathrm{m}$ ) form and the corresponding loading rate was $5 \mathrm{pN} / \mathrm{s}$.

The elastic modulus was calculated using the Hertz model. ${ }^{29}$ The apparent elastic modulus, $E$, is

$$
E=3\left(1-v^{2}\right) F /(4 \operatorname{Id} \sqrt{\operatorname{Id} \times R}),
$$

where $R$ is the bead radius, $F$ is the force, $\nu(=0.4)$ is the Poisson ratio, and Id is the indentation, calculated as (see inset Fig. 1(b))

$\mathrm{Id}=\mathrm{SD}-\mathrm{BD}$.

The elastic modulus formula [Eq. (1)] can be expressed as a function of indentation, Id, as

$$
F=E\left[(4 \sqrt{r}) /\left(3\left(1-v^{2}\right)\right] \mathrm{Id}^{2 / 3}\right.
$$

Linearizing the preceding equation in terms of Id leads to

$$
F^{3 / 2}=E^{3 / 2} \times C \times \mathrm{Id},
$$

where $C$ is a constant including all the other constants in Eq. (3). By fitting linearly the $F^{3 / 2}$ curve, one can easily determine the value of the elastic modulus $E$.

\subsection{Immunostaining and Confocal Microscopy Imaging}

Cells were fixed in $4 \%$ paraformaldehyde containing $0.15 \%$ picric acid in PBS, saturated with $0.1 \mathrm{M}$ glycine, permeabilized with $0.1 \%$ Triton $\mathrm{X}-100$, saturated with $0.5 \%$ BSA in PBS (all from Sigma-Aldrich, St. Louis, Missouri) and then incubated for 40 min with Alexa Fluor 488 phalloidin (Invitrogen, Life Technologies, Gaithersburg, Maryland). Cells were further washed, before mounting in Vectashield (VECTOR Laboratories, Burlingame, California) incubated with 4',6-diamidino-2phenylindole $(1: 500)$ in PBS for $5 \mathrm{~min}$. All the incubations were performed at room temperature $\left(20^{\circ} \mathrm{C}\right.$ to $\left.22^{\circ} \mathrm{C}\right)$. Cells were examined using a Leica DMIRE2 confocal microscope (Leica Microsystems GmbH, Germany) equipped with DIC and fluorescence optics, diode laser $405 \mathrm{~nm}, \operatorname{Ar} / \mathrm{ArKr} 488 \mathrm{~nm}$, and $\mathrm{He} / \mathrm{Ne} 543 / 594 \mathrm{~nm}$ lasers. The fluorescence images $(1024 \times$ 1024 pixels) were collected with a $60 \times$ and 1.3 NA oil immersion objective.

\section{Results}

\subsection{Stiffness at Different Subcellular Regions}

A wide range of approaches like AFM, optical starching, and micropipette aspiration have been used to study viscoelastic properties of cellular subregions. ${ }^{27,35-37}$ We used OT for the first time to study cell stiffness at three different cell locations. All three cell lines in isolated condition are indented at the three subregions as indicated in Fig. 2(a). The results [Fig. 3(a) and Table 1] show a descending trend in elastic modulus from nuclear region toward the leading edge. All three cell lines exhibit higher stiffness at the center (L1) than the intermediate position (L2) yet having higher value than position L3 near the leading edge. The elastic modulus values at the three locations

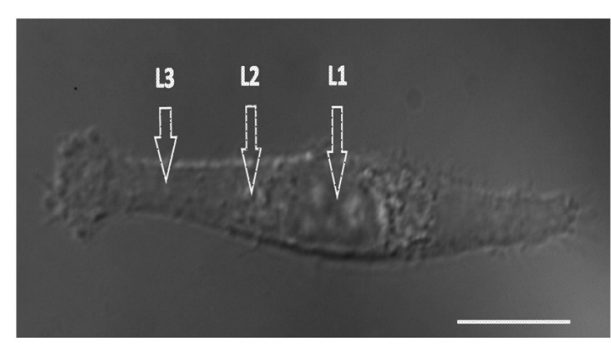

(a)

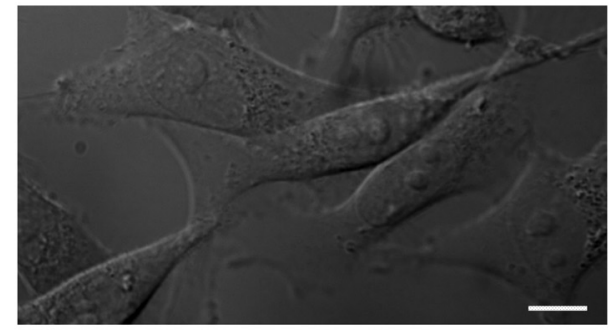

(b)

Fig. 2 Differential interference contrast (DIC) image of MDA-MB-231 cells cultured on glass substrate. Cell is categorized as (a) isolated cell: when there is no interaction with any other cell and (b) connected cell: when the cell interacts with two or more cells. Each cell is indented at three locations: L1-above nucleus, L2-intermediate position (cytoplasm), and L3-near the leading edge (a). Scale bars are $10 \mu \mathrm{m}$. 


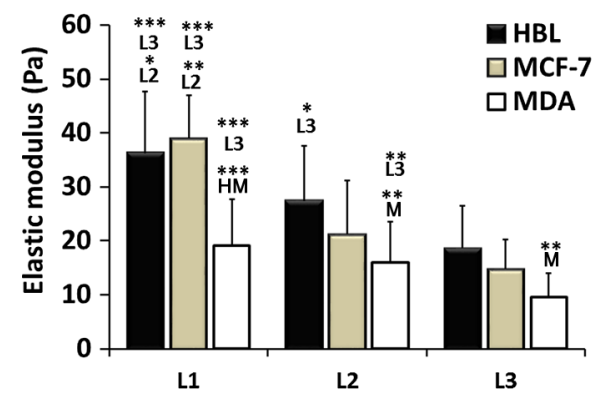

(a)

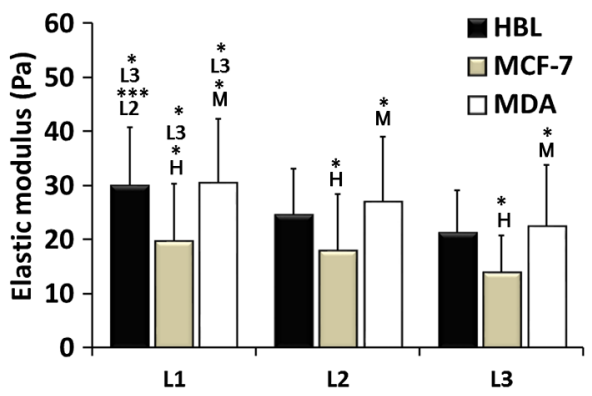

(b)

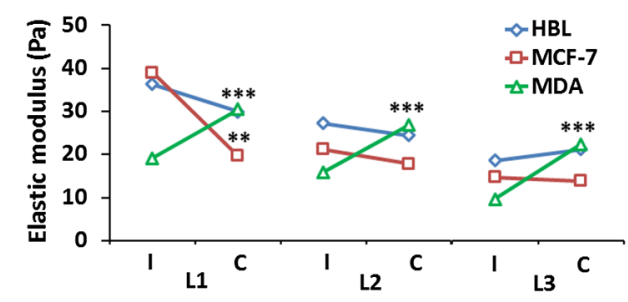

(c)

Fig. 3 (a) Isolated cells: all the three cell lines have descending character for stiffness, higher at the center and decreasing toward the leading edge. (b) Connected cells: stiffness decreases from nuclear region to the leading edge. Cell lines exhibit the same stiffness in the cytoplasm and leading edge indicating that cell cytoskeleton organization during interactions becomes more dynamic. MDA-MB-231 shows the same stiffness as HBL-100. (c) Elastic moduli variation in isolated (I) and connected (C) conditions in nuclear (L1), cytoplasm (L2), and leading edge (L3) regions. MDA-MB-231 exhibits a more prominent variation in connected conditions as compared to HBL-100 and MFC-7. The symbols represent H: HBL-100 and M: MCF-7. ( $t$-test: ${ }^{*} p<0.05,{ }^{* \star} p<0.01$, and ${ }^{* \star *} p<0.001$ ).

Table 1 Elastic modulus measurements ( \pm standard error of the mean) for all three cell lines measured in isolated and connected conditions in three different cellular subregions (L1, L2, and L3).

\begin{tabular}{|c|c|c|c|c|c|c|c|c|c|c|c|c|}
\hline & \multicolumn{6}{|c|}{ Cell stiffness at cellular subregions in isolated condition } & \multicolumn{6}{|c|}{ Cell stiffness at cellular subregions in cell-cell contact condition } \\
\hline & \multicolumn{2}{|c|}{ HBL-100 } & \multicolumn{2}{|c|}{ MCF-7 } & \multicolumn{2}{|c|}{ MDA-MB-231 } & \multicolumn{2}{|c|}{ HBL-100 } & \multicolumn{2}{|c|}{ MCF-7 } & \multicolumn{2}{|c|}{ MDA-MB-231 } \\
\hline & $E(\mathrm{~Pa})$ & $n$ & $E(\mathrm{~Pa})$ & $n$ & $E(\mathrm{~Pa})$ & $n$ & $E(\mathrm{~Pa})$ & $n$ & $E(\mathrm{~Pa})$ & $n$ & $E(\mathrm{~Pa})$ & $n$ \\
\hline L1 & $36 \pm 11$ & 10 & $39 \pm 8$ & 10 & $19 \pm 9$ & 14 & $30 \pm 11$ & 29 & $20 \pm 11$ & 15 & $31 \pm 12$ & 14 \\
\hline L2 & $27 \pm 10$ & & $21 \pm 10$ & & $16 \pm 8$ & & $25 \pm 9$ & & $18 \pm 11$ & & $27 \pm 12$ & \\
\hline L3 & $19 \pm 8$ & & $16 \pm 6$ & & $10 \pm 4$ & & $21 \pm 8$ & & $14 \pm 7$ & & $23 \pm 11$ & \\
\hline
\end{tabular}

are statistically significant and in agreement with studies carried out by other groups using different biophysical methods. ${ }^{27,36,37}$

Stiffness values for single cells indented at the center (L1) show that the most aggressive cells (MDA-MB-231) are less stiff as compared to HBL-100 and MCF-7. These values agree with other results reported in the literature, showing that metastatic cells are softer as compared to their noninvasive counter parts. ${ }^{4,18,27,35,38}$ The HBL-100 and MCF-7 cells are twofold stiffer than MDA-MB-231 at the nuclear region (L1) and can be used to distinguish between cells with different aggressiveness levels. These results also act as first test for the feasibility of our successive experiments on cell stiffness as a function of cell subregions and of cell-cell interaction.

When comparing different cellular subregions, for HBL-100 cells, the stiffness at the nuclear region is $25 \%$ higher than that corresponding to the cytoplasm region and $47 \%$ higher than that corresponding to the leading edge. For MCF-7 cells, the stiffness decreases as $46 \%$ and $59 \%$, whereas for MDA-MB231 it diminishes by $16 \%$ and $47 \%$, respectively, for cytoplasm and the leading edge. All three cell lines have significantly different stiffness values at the nuclear region and the leading edge, showing a larger contribution of the nucleus toward mechanical architecture. ${ }^{37}$ Our results stress that for single-cell force measurement, the region of interest affects mechanical observation and hence should be considered for mechanical studies of the cells.

\subsection{Cell-Cell Contact Alters Cell Stiffness}

To investigate the stiffness variation during interactions with neighboring cells, we analyzed cells from three different cell lines in contact conditions [Fig. 2(b)]. Prominent variation in stiffness was observed as summarized in Table 1 and shown in Fig. 3(b). Analyzing the cellular regional variation, one can note that the cell stiffness of HBL-100 and MCF-7 cells decreases by 
$(17 \%$ and $10 \%)$ and $(30 \%$ and $30 \%)$, while for MDA-MB-231 it decreases by $13 \%$ and $26 \%$ at the cytoplasm and leading edge regions as compared with the nuclear region. Also, in contact conditions, cell stiffness at the leading edge has a significantly lower value than at the nuclear region. However, when compared to the isolated condition, one observes that the stiffness of the MD-MB-231 cells in connected condition increases and becomes comparable with that of the HBL-100 cells for all the three locations [Fig. 3(b)]. This behavior is opposite to the behavior of the other two cell lines. Comparing the stiffness values corresponding to regional locations in isolated and connected conditions, we find a decreasing trend for HBL-100 and MCF-7, whereas MDA-MB-231 stiffness is increased, as shown in Fig. 3. For HBL-100 and MCF-7, stiffness at nuclear region decreases by $17 \%$ and $49 \%$, respectively. MDA-MB-231 responds differently to cell neighboring and becomes stiffer in contact condition. Stiffness increases by $39 \%, 41 \%$, and $57 \%$ in L1, L2, and L3 locations, respectively. The higher increment at the leading edge in contact condition suggests that MD-MB-231 orchestrates more cytoskeletal components at the leading edge and applies greater force on the membrane.

HBL-100 and MDA-MB-321 cells do not express E-cadherins, which are involved in cell-cell tight junctions, whereas MCF-7 does express them; hence, they experience different interaction mechanisms. ${ }^{27,35}$ Furthermore, HBL-100 (nontumorigenic) and MCF-7 (tumorigenic) are noninvasive, but MDA-MB-321 is invasive; therefore, their response to the interacting cells might be different. Lee et al. ${ }^{35}$ have shown that MDA-MB-231 cells are more mobile in the active interaction environment of MCF-10A (nontumorigenic epithelial cell line and normal counterpart of MCF-7) monolayer as compared to noninvasive MCF-7 cells. They showed that invasive cells (MDA-MB-321) are softer and more sensitive to physical forces, if applied on the lateral surfaces of the cells. Our results show that MDA-MB-231 cells, though softer in isolated conditions, feel stressed in interacting environment whereas the MCF-7 and HBL-100 turns softer during cell-cell interactions, in agreement with the results reported by Kristal-Muscal et al. ${ }^{38}$ They demonstrated that the cells with metastatic potential (MDA-MD-231) generate greater forces despite their soft character as compared to low metastatic potential cell (MDAMB-468) and benign cells (MCF-10A), which do not indent the substrate at all. ${ }^{33}$

\section{Discussion}

Cancer cell metastasis formation is a complicated mechanism. The evidence indicates that in the cancer microenvironment, ECM becomes stiffer as compared to its normal state, but cells behave softer ${ }^{39}$ and exhibit increased actomyosin cortex contractility. ${ }^{33,34}$ Furthermore, some studies show that by altering the mechanics of cell microenvironment, cancerous cells may act as normal ones. ${ }^{14,15}$

Interesting results on the effect of cell-cell contact on cell motility and cell adhesion have been reported for MDA-MB231 cells. ${ }^{35}$ These results indicate that the motility of MDAMB-231 cells, which are mechanically soft, is dramatically enhanced when they are surrounded by breast epithelial MCF$10 \mathrm{~A}$ cells, which are mechanically stiff, compared with singlecell motility within a cluster of MDA-MB-231 cells (high density) or single MDA-MB-231 cells (low density) in the absence of non-neoplastic MCF-10A cells. Another interesting result is the cell-substrate contact/interaction effect on the cell's ability to apply forces, ${ }^{38}$ showing that cells of higher metastatic potential develop stronger forces on gels stiff enough to provide grip handles yet soft enough to indent, whereas benign cells did not indent substrates at all.

Our work was focused on cell stiffness as a marker to distinguish between cells at different stages of cancer progression. It is known that the values obtained for the cell stiffness might vary depending on the measurement method; therefore, employing complementary techniques (e.g., OT and AFM) increases the reliability. ${ }^{30}$ Using OT, we show that for the isolated cell condition, the metastatic cells are softer than tumorigenic and nonneoplastic cells and cell stiffness decreases from the nuclear region toward the cell edge for all the three cell models. The results are confirmed by measurements using peak force AFM in our recent work ${ }^{30}$ and in that of another group. ${ }^{31}$ These results are in line with the general observation that malignant cells are softer than normal cells across a range of different cancers. ${ }^{13}$ However, our results appear partially in contradiction with those obtained by Guo et al. ${ }^{27}$ Using AFM to measure cell stiffness, they report that metastatic cells are slightly stiffer than tumorigenic cells and only minor or negligible differences in stiffness differentiate the cellular regions. In our opinion, the difference might be attributed to the differences between the cell models (only the metastatic cells MDA-MB-231 are identic) and the difference between the indentation regimes (indentation force, indentation range, and loading rate). Moreover, the experiments by Guo et al. were performed at room temperature, while ours were conducted at the physiological temperature $\left(37^{\circ} \mathrm{C}\right)$. It is well known that temperature strongly influences cell health, behavior, and properties (mechanical properties included).

We found that for the metastatic cells MDA-MB-231 cell neighboring induces a significant increase in stiffness. This trend is confirmed for all three locations of measurements, above the nucleus, the cytoplasm, or near the cell edge. The cell stiffness for the other two cell lines (HBL-100 and MCF-7) decreases significantly for the nuclear region and shows minor changes for the other two regions. To our knowledge, this result points to the importance of cell environment when defining cell stiffness. The result is partially in contradiction with that showing the microenvironment condition has little or no effect on immortal and metastatic cells reported by Guo et al., probably due to the same aforementioned factors for the cell stiffness variation with the cell regions. Trying to associate the changes of cell stiffness with the cytoskeleton rearrangements, we analyzed F-actin organization in isolated and connected cells. Some sample images using immunofluorescence confocal microscopy are shown in Fig. 4 for HBL-100 and MDA-MB-231 cells. While for the HBL-100 cells, we have not observed a significant difference between the actin fibers organization in isolated and connected cells, the actin stress fibers seem to be enforced for the connected MDA-MB-231 cells with respect to the isolated cells. This result has to be further confirmed by a more thorough analysis of the indentation regions using higher spatial resolution or combined techniques. In fact, combining immunofluorescence confocal microscopy and peak-force modulated AFM, Calzado-Martin et al. recently showed that actin stress fibers are present at apical regions of healthy cells, while in tumorigenic cells they appear only at basal regions, where they cannot contribute to stiffness as probed by AFM. These results substantiate that actin stress fibers provide a dominant contribution to stiffness in healthy 


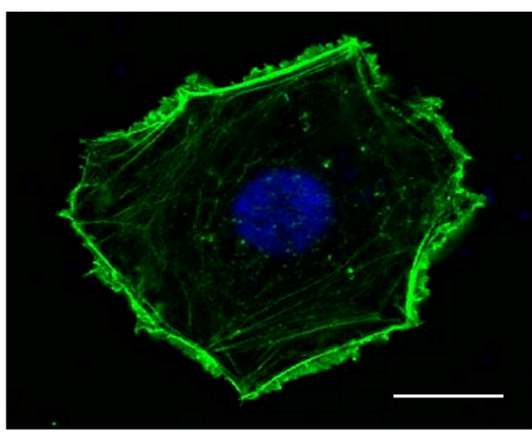

(a)

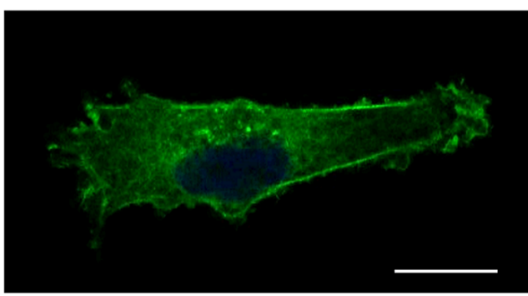

(c)

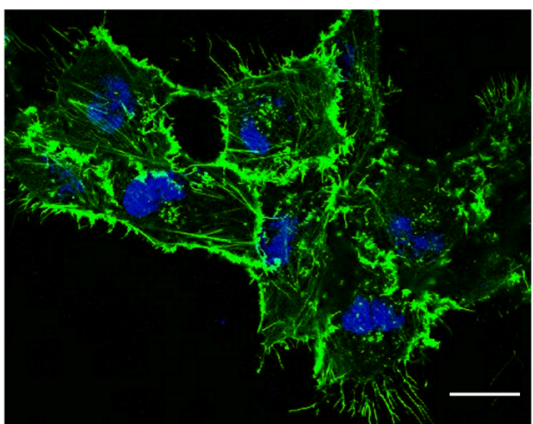

(b)

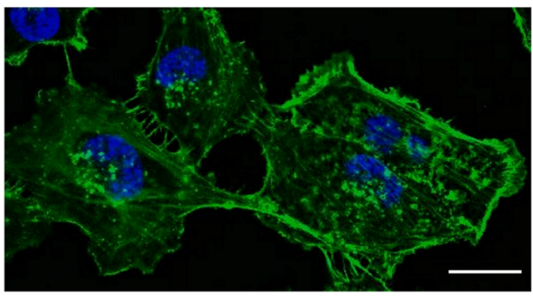

(d)

Fig. 4 Immunofluorescence images for F-actin networks in HBL-100 cell [(a) isolated and (b) connected] and MDA-MB-231 cells [(c) isolated and (d) connected]. Scale bars are $10 \mu \mathrm{m}[(\mathrm{a})$ and (c)] and $20 \mu \mathrm{m}[(\mathrm{b})$ and (d)].

cells, while the elasticity of tumorigenic cells appears not predominantly determined by these structures. The combination of these two techniques could be applied in the future to investigate also the differences in actin fibers organization for isolated and connected cells.

\section{Conclusions}

We report an OT approach to investigate on the change of the cell stiffness determined by cell neighboring, and we show that the metastatic cells are those which are influenced the most. By indenting cells axially on the top, we demonstrate that stiffness measurements are sensitive to the cellular subregions as well as the interacting microenvironment. Altogether, our results point to the importance of cell microenvironment when defining cell stiffness. In future studies, we will apply this technique also to investigate effect of ECM on cell-cell interaction and to study the related mechanotransduction pathways. More rigorous mechanical studies on cancer cells corresponding to different range of forces and different microenvironmental conditions may further unravel aspects of cancer progression and metastasis formation. Models of cell cytoplasm behavior and reorganization, like the poroelastic material model, ${ }^{40}$ will help a better understanding of the relation between the cytoskeleton structure and cell stiffness. Cells in 3-D rather than 2-D cell neighboring would be very interesting to be investigated and probably the results will show more variations in terms of cell stiffness. Engineering 3-D cell structures is already a reality, ${ }^{41}$ but measuring cell stiffness is unfortunately still impossible with OT or AFM in these conditions. Different probing approaches should be developed, which represents the challenge for the future.

\section{Acknowledgments}

We would like to gratefully acknowledge the Applied Physics Section of the International Centre for Theoretical Physics, Trieste, for partial financial support to build the OT setup, and partial financial support by the European Research Council (ERC) Ideas Program through a senior grant to Giacinto Scoles, titled Molecular Nanotechnology for Life Science Applications: Quantitative Interactomics for Diagnostics, Proteomics and Quantitative Oncology (MONALISA QUIDPROQUO) Grant No. 269051.

\section{References}

1. D. Hanahan and R. A. Weinberg, "The hallmarks of cancer," Cell 100(1), 57-70 (2000).

2. D. Hanahan and R. A. Weinberg, "Hallmarks of cancer: the next generation," Cell 144(5), 646-674 (2011).

3. R. Siegel et al., "Cancer statistics, 2014," CA: Cancer J. Clin. 64(1), 9-29 (2014)

4. S. Suresh, "Biomechanics and biophysics of cancer cells," Acta Mater. 55(12), 3989-4014 (2007).

5. G. P. Gupta and J. Massagué, "Cancer metastasis: building a framework," Cell 127(4), 679-695 (2006).

6. H. Huang et al., "Cell stiffness and receptors: evidence for cytoskeletal subnetworks," Am. J. Physiol. Cell Physiol. 288(1), C72-C80 (2005).

7. I. Schoen, B. L. Pruitt, and V. Vogel, "The Yin-Yang of rigidity sensing: how forces and mechanical properties regulate the cellular response to materials," Аnnu. Rev. Mater. Res. 43, 589-618 (2013).

8. Q. Wen and P. A. Janmey, "Effects of non-linearity on cell-ECM interactions," Exp. Cell. Res. 319(16), 2481-2489 (2013).

9. T. R. Geiger and D. S. Peeper, "Metastasis mechanisms," $B B A$ 1796(2), 293-308 (2009).

10. M. Makale, "Cellular mechanobiology and cancer metastasis," Birth Defects Res. Part C 81(4), 329-343 (2007).

11. N. Guz et al., "If cell mechanics can be described by elastic modulus: study of different models and probes used in indentation experiments," Biophys. J. 107(3), 564-575 (2014).

12. P. Schedin and A. Elias, "Multistep tumorigenesis and the microenvironment," Breast Cancer Res. 6(2), 93-101 (2004).

13. C. T. Mierke, "The fundamental role of mechanical properties in the progression of cancer disease and inflammation," Rep. Prog. Phys. 77(7), 076602 (2014). 
14. M. J. Bissell and W. C. Hines, "Why don't we get more cancer? A proposed role of the microenvironment in restraining cancer progression," Nat. Med. 17(3), 320-329 (2011).

15. D. E. Ingber, "Can cancer be reversed by engineering the tumor microenvironment?” Semin. Cancer Biol. 18(5), 356-364 (2008).

16. D. Wirtz, K. Konstantopoulos, and P. C. Searson, "The physics of cancer: the role of physical interactions and mechanical forces in metastasis," Nat. Rev. Cancer 11(7), 512-522 (2011).

17. T. Risler, "Focus on the physics of cancer," New J. Phys. 17(5), 055011 (2015).

18. B. D. Hoffman and J. C. Crocker, "Cell mechanics: dissecting the physical responses of cells to force," Annu. Rev. Biomed. Eng. 11, 259-288 (2009).

19. G. Cheng et al., "Micro-environmental mechanical stress controls tumor spheroid size and morphology by suppressing proliferation and inducing apoptosis in cancer cells," PLoS One 4(2), e4632 (2009).

20. B. Ladoux and A. Nicolas, "Physically based principles of cell adhesion mechanosensitivity in tissues," Rep. Prog. Phys. 75(11), 116601 (2012).

21. J. Fu et al., "Mechanical regulation of cell function with geometrically modulated elastomeric substrates," Nat. Methods 7(9), 733-736 (2010).

22. R. S. Gieni and M. J. Hendzel, "Mechanotransduction from the ECM to the genome: are the pieces now in place?" J. Cell. Biochem. 104(6), 1964-1987 (2008).

23. D. E. Jaalouk and J. Lammerding, "Mechanotransduction gone awry," Nat. Rev. Mol. Cell Biol. 10(1), 63-73 (2009).

24. L. P. Ivers et al., "Dynamic and influential interaction of cancer cells with normal epithelial cells in 3D culture," Cancer Cell Int. 14(1), 108 (2014).

25. S. Hu et al., "Manipulating cell adhesions with optical tweezers for study of cell-to-cell interactions," J. Biomed. Nanotechnol. 9(2), 281-285 (2013).

26. X. Gou et al., "Applying combined optical tweezers and fluorescence microscopy technologies to manipulate cell adhesions for cell-to-cell interaction study," IEEE Trans. Biomed. Eng. 60(8), 2308-2315 (2013).

27. X. Guo et al., "The effect of neighboring cells on the stiffness of cancerous and non-cancerous human mammary epithelial cells," New J. Phys. 16(10), 105002 (2014).

28. K. Kamińska et al., "The role of the cell-cell interactions in cancer progression," J. Cell. Mol. Med. 19(2), 283-296 (2015).
29. S. Nawaz et al., "Cell visco-elasticity measured with AFM and optical trapping at sub-micrometer deformations," PLoS One 7(9), e45297 (2012).

30. G. Coceano et al., "Investigation into local cell mechanics by atomic force microscopy mapping and optical tweezer vertical indentation," Nanotechnology 27(6), 065102 (2015).

31. A. Calzado-Martin et al., "Effect of actin organization on the stiffness of living breast cancer cells revealed by peak-force modulation atomic force microscopy," ACS Nano 10(3), 3365-3374 (2016).

32. C. C. De Fromentel et al., "Epithelial HBL-100 cell line derived from milk of an apparently healthy woman harbours SV40 genetic information," Exp. Cell Res. 160(1), 83-94 (1985).

33. M. S. Yousafzai et al., "Substrate-dependent cell elasticity measured by optical tweezers indentation," Opt. Lasers Eng. 76, 27-33 (2016).

34. F. Gittes and C. F. Schmidt, "Interference model for back-focal-plane displacement detection in optical tweezers," Opt. Lett. 23(1), 7-9 (1998).

35. M.-H. Lee et al., "Mismatch in mechanical and adhesive properties induces pulsating cancer cell migration in epithelial monolayer," Biophys. J. 102(12), 2731-2741 (2012).

36. J. Guck et al., "Optical deformability as an inherent cell marker for testing malignant transformation and metastatic competence," Biophys. $J$. 88(5), 3689-3698 (2005).

37. F. Guilak, J. R. Tedrow, and R. Burgkart, "Viscoelastic properties of the cell nucleus," Biochem. Biophys. Res. Commun. 269(3), 781-786 (2000).

38. R. Kristal-Muscal, L. Dvir, and D. Weihs, "Metastatic cancer cells tenaciously indent impenetrable, soft substrates," New J. Phys. 15(3), 035022 (2013).

39. P. Katira, R. T. Bonnecaze, and M. H. Zaman, "Modeling the mechanics of cancer: effect of changes in cellular and extra-cellular mechanical properties," Front. Oncol. 3, 145 (2013).

40. E. Moeendarbary et al., "The cytoplasm of living cells behaves as a poroelastic material," Nat. Mater. 12(3), 253-261 (2013).

41. J. W. Haycock, "3D cell culture: a review of current approaches and techniques," in 3D Cell Culture: Methods and Protocols, Methods in Molecular Biology, pp. 1-15, Humana Press, New York (2011). 